\title{
Multi-layer spectral clustering approach to intentional islanding in bulk power systems
}

\author{
Faycal ZNIDI ${ }^{1}$ (i), Hamzeh DAVARIKIA ${ }^{2}$, Kamran IQBAL $^{1}$, \\ Masoud BARATI ${ }^{3}$
}

MPCE

\begin{abstract}
Intentional controlled islanding (ICI) is a final resort for preventing a cascading failure and catastrophic power system blackouts. This paper proposes a controlled islanding algorithm that uses spectral clustering over multilayer graphs to find a suitable islanding solution. The multi-criteria objective function used in this controlled islanding algorithm involves the correlation coefficients between bus frequency components and minimum active and reactive power flow disruptions. Similar to the previous studies, the algorithm is applied in two stages. In the first stage, groups of coherent buses are identified with the help of modularity clustering using correlation coefficients between bus frequency components. In the second stage, the ICI solution satisfying bus coherency with minimum active and reactive power flow disruptions is determined by grouping all nodes using spectral clustering on the multi-
\end{abstract}

CrossCheck date: 28 April 2019

Received: 24 September 2018/Accepted: 28 April 2019/Published online: 27 July 2019

(C) The Author(s) 2019

$\triangle$ Faycal ZNIDI

fxznidi@ualr.edu

Hamzeh DAVARIKIA

hdavar1@1su.edu

Kamran IQBAL

kxiqbal@ualr.edu

Masoud BARATI

masoud.barati@pitt.edu

1 Department of Systems Engineering, University of Arkansas at Little Rock, Little Rock, AR, USA

2 Department of Electrical and Computer Engineering, Louisiana State University, Baton Rouge, LA, USA

3 Department of Electrical and Computer Engineering, University of Pittsburgh, Pittsburgh, PA, USA layer graph. Simulation studies on the IEEE 39-bus test system demonstrate the effectiveness of the method in determining an islanding solution in real time while addressing the generator coherency problem.

Keywords Constrained spectral clustering, Controlled islanding, Bus coherency, Multi-layer graph, Normalized spectral clustering

\section{Introduction}

Intentional controlled islanding (ICI) has been proposed as a corrective measure of last resort to split the power system into several sustainable islands and prevent cascading outages. Most approaches to islanding aim to find, as a primary objective, electromechanically stable islands with minimum load shedding. To find a reasonably good islanding solution, all subsystems must satisfy some constraints such as power flow disruption, generator coherency, transient stability, etc. [1].

Traditionally, the islanding problem has been solved using combinatorial optimization approaches. The inclusion of reactive power or voltage in the constraints leads to a mixed integer nonlinear program (MINLP) problem, which is, in general, more difficult to be solved than nonlinear programming problem (NPP) and mixed integer linear programming (MILP) problem [1, 2]. Therefore, linear DC power flow has often been used in literature resulting in a MILP problem that promises a better computational burden. Additionally, some other methods consider only the active power in system partitioning. As an example, in [2], it is claimed that local reactive power compensators can be used to compensate reactive power imbalance. In [3], a MILP-based splitting strategy is 
proposed to manage energy production and demand. In this methodology, the reactive power is viewed as a local issue and can be handled with local reactive power compensators and only active power is considered in the splitting scheme. However, the reactive power plays a significant role in supporting the voltage profile, and a significant mismatch of the reactive power supply and demand causes high or low voltage conditions within islands. In [4], the MILPbased optimization method for controlled islanding disregards the generator coherency constraint, which is one of the most important requirements in the islanding solution. The optimization-based islanding algorithms are proposed in $[5,6]$, aiming to find the boundaries of electric islands. Utilizing the mathematical programming for islanding solution requires different sets of constraints to ensure the island integrity and feasibility, including unlimited to power balanced, connectivity, and operation constraints. On the other hand, the graph-based islanding solutions automatically satisfy the connectivity constraints, since the solution is sought through the minimum cuts in the graph.

In [7], the constrained spectral clustering is used to find islanding boundary with minimum power flow disruption. In [8], a binary particle swarm optimization (BPSO) seeking Pareto non-dominated solutions algorithm is presented to find islands containing coherent generator groups with minimum power imbalances. As BPSO is a stochastic evolutionary algorithm, its multiple runnings are needed to determine if the results are consistent. In [9], a two-step spectral clustering controlled islanding algorithm is introduced, while using generator coherency as the sole constraint with minimum active power flow disruption objective to find a suitable ICI solution. In [10, 11], the authors present an islanding scheme with minimum active power flow disruption using a constrained spectral embedded clustering technique, while satisfying the generator coherency constraints. However, these techniques disregard the effects of the bus voltage magnitude and reactive power, which have a substantial impact on the dynamic coupling. In [12], a methodology based on dynamic frequency deviations of both generator and nongenerator buses, with respect to the system nominal frequency is presented. Overall the center of inertia concept has shown its advantages in various applications.

While there is an in-depth treatment of individual topics such as generator coherency and the active and reactive power graph based models in the literature, there is a dearth of information regarding these multiple topics in a single model [13-16].

In this paper, a multi-layer graph spectral clustering controlled islanding (M-SCCI) algorithm for ICI solution of power systems is presented. In the first stage of the algorithm, the frequency similarity of buses, the active power flow between buses, and the reactive power flow between buses construct three different layers of the multilayer graph. The frequency similarity among each pair of buses is evaluated using the correlation among the bus frequency components. To determine the number of islands, the modularity clustering is applied to the layer containing frequency similarities among buses, which results in " $k$ " numbers of coherent buses or coherent groups of generators. The number of " $k$ " cluster outcomes of this grouping serves as the input in the second stage of the M-SCCI algorithm that identifies islanding boundaries with minimum active and reactive power flow disruption. This technique is based on a multi-layer graph, whose common vertex set represents the buses, and the edges on individual layers represent power system attributes that reflect the similarities among the buses in term of the various modalities. These modalities include: (1) frequency correlation coefficient between buses; (2) real power flow disruption; (3) reactive power flow disruption.

\section{Graph theory approach to controlled islanding problem}

\subsection{Multi-layer graph models of power systems}

An electrical network is undirected graph $G(V, E, w)$ where each element $v_{i} \in V$ is either a substation or a transformer, the edge $e_{i, j}=\left(v_{i}, v_{j}\right) \in E$ is a physical cable between two nodes $[17,18]$, and $w$ is the associated edge weight. A multi-layer graph $G$ consists of $M$ distinct graph layers $G_{i}, i=1,2, \ldots, M$, where each distinct layer $G_{i}=$ $\left\{V, E_{i}, w_{i}\right\}$ is a undirected and weighted graph composed over a common vertex set $V$ and particular edge set $E_{i}$ with associated weights $w_{i}$ [19]. The sets comprising the graph assume interest from an operational and physical point of view. The individual layers characterize specific relationships among entities, such as the frequency similarity associated with each pair of the island buses, and the active and reactive power flow disruptions.

A generic representation of the three-layer graph in power system is depicted in Fig. 1, where the first, second and third layers are associated to the frequency similarity, active power flow, and reactive power flow, respectively. These layers have the same nodes which represent the buses in power networks, while the edges are associated to the frequency similarity between buses in layer 1 , the active power flow between buses in layer 2 , and the reactive power flow between buses in layer 3 . While the first layer is a full weighted graph (all the nodes are connected with each other), the other layers have the same edges as physical lines in power networks. 


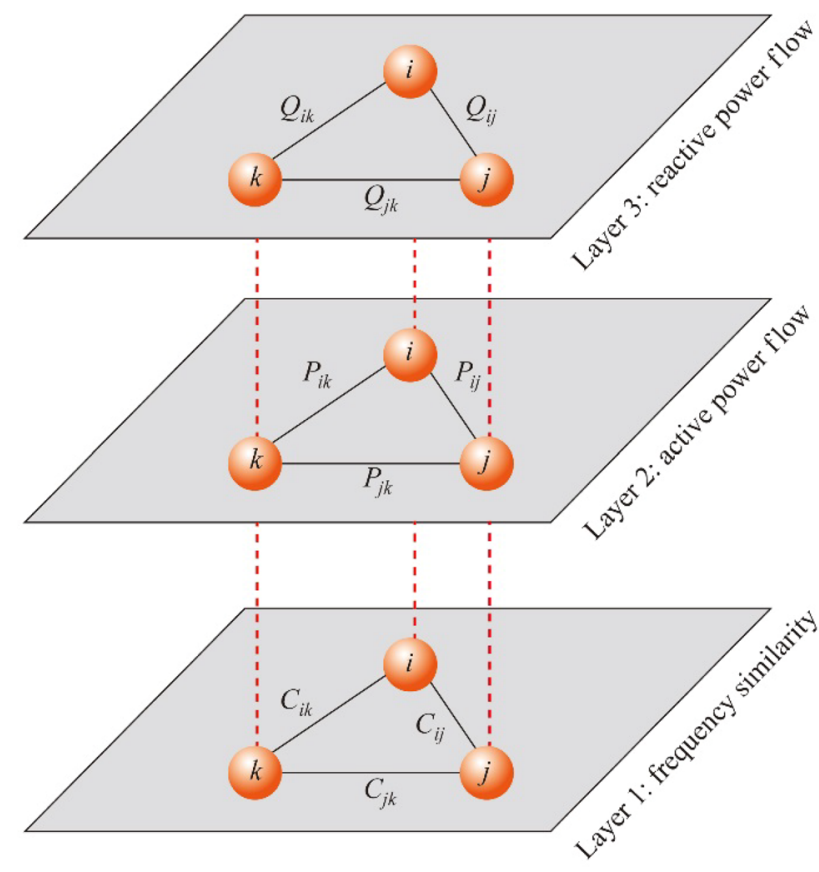

Fig. 1 Three-layer graph of power networks

\subsection{Dynamic generator coherency}

Following a sudden disturbance on the power grid, the dynamic response of individual generators can be determined by phase angle dissimilarity at the buses close to the generator. The frequencies that represent the dynamic response of every generator after grid disturbances can be defined as:

$s_{i, j}=\int_{0}^{T}\left(\Delta \theta_{i}(t)-\Delta \theta_{j}(t)\right) \mathrm{d} t$

where $\theta_{i}$ and $\theta_{j}$ are the phase angles at bus $i$ and bus $j$, respectively; $T$ is the observation time; and $s_{i, j}$ is the dissimilarity index between bus $i$ and bus $j$.

The amount of energy observed or delivered by generators in the power system can be reflected by their speed deviations [19]. Therefore, analyzing these frequencies which represent the dynamic response of generators following a disturbance can be helpful for coherency determination. These frequency components can be extracted using the discrete Fourier transform (DFT) as follows:

$$
\begin{aligned}
& F_{i}(f)=\int_{0}^{N-1} \omega_{i}(k) \mathrm{e}^{-\mathrm{j} \frac{2 \pi f k}{N}} \mathrm{~d} k \quad f=0,1, \ldots, N-1 \\
& \omega_{i}(k)=\frac{\theta_{i}(k)-\theta_{i}(k-1)}{\Delta t}
\end{aligned}
$$

where $\omega_{i}(k)$ is the angular velocity of generator $i$ at time instant $k ; F_{i}(f)$ is the Fourier transform of the angular speed; $N$ is the number of samples in the waveform; and $\Delta t$ is the time interval between two consecutive samples, which holds constant throughout simulations.

The vector-space $\boldsymbol{F}_{i}=\left[F_{i}(1), F_{i}(2), \ldots, F_{i}(N)\right]^{\mathrm{T}}$ and $N_{B} \times N$ dimension matrix $\boldsymbol{F}$ are formed as:

$\boldsymbol{F}=\left[\begin{array}{llllll}\boldsymbol{F}_{1} & \boldsymbol{F}_{2} & \ldots & \boldsymbol{F}_{i} & \ldots & \boldsymbol{F}_{N_{B}}\end{array}\right]^{\mathrm{T}}$

where $N_{B}$ is the total number of buses in the power grid.

The phase angle and the amplitude of each frequency component in the angular velocity signal can be extracted by using the DFT. Therefore, the correlation of the phase angle oscillation of generator/non-generator buses can reveal the coherency of oscillations related to generators, which will be discussed in the next section.

\subsection{Correlation coefficient similarity matrix}

Pearson product-moment correlation coefficient $C_{C}$ is a popular metric used to evaluate the strength of the association between two variables $[5,16]$. The $C_{C}$ ranges between -1.0 and +1.0 and quantifies the direction and strength of the linear association between two multi-dimensional random variables. In connection with power systems, this factor represents the association between two different electrical buses, as shown in (5).

$$
C_{C, i j}=\frac{\sum_{f=1}^{n}\left(F_{i}(f)-F_{i, a v g}\right)\left(F_{j}(f)-F_{j, a v g}\right)}{\sqrt{\sum_{f=1}^{n}\left(F_{i}(f)-F_{i, a v g}\right)^{2} \times \sum_{f=1}^{n}\left(F_{j}(f)-F_{j, a v g}\right)^{2}}}
$$

where $C_{C, i j}$ is the correlation coefficient among buses $i$ and $j$, a larger $C_{C, i j}$ indicates a stronger connection or higher coherency between bus $i$ and bus $j ; n$ is the number of frequency components; and $F_{i, a v g}$ is the average of the frequency components of bus $i$ in the domain of inter-area oscillation modes. We define the correlation coefficient similarity matrix as $\boldsymbol{M}_{C C S M}$, whose components are equal to $C_{C, i j}, i, j \in N_{B}$, namely,

$\boldsymbol{M}_{C C S M}=\left[\begin{array}{cccc}C_{C, 11} & C_{C, 12} & \ldots & C_{C, 1 N_{B}} \\ C_{C, 21} & C_{C, 22} & \ldots & C_{C, 2 N_{B}} \\ \vdots & \vdots & & \vdots \\ C_{C, N_{B} 1} & C_{C, N_{B} 2} & \ldots & C_{C, N_{B} N_{B}}\end{array}\right]$

\subsection{Reactive power similarity matrix}

Generally, in a power system, the voltage and the frequency are controlled by reactive power and active power, respectively. Therefore, considering reactive power and active power simultaneously in the islanding problem would result in more stable islands in terms of frequency and voltage. In order to accomplish the aforementioned goal, the minimum power flow disruption, as shown in (7), 
can be utilized for controlled islanding as the objective function.

$\min _{V_{1}, V_{2} \subset V}\left(\sum_{i \in V_{1}, j \in V_{2}}\left|Q_{i j}\right|\right)$

where $Q_{i j}$ is the reactive power flow between bus $i$ and bus $j$. The controlled islanding problem with the above objective function can be transformed into a graph-cut problem by defining a squared $N_{B} \times N_{B}$ adjacency matrix with elements $\left|Q_{i j}\right|$. Accordingly, a reactive power graph similarity matrix $\boldsymbol{M}_{Q}$ is defined as:

$M_{Q, i j}= \begin{cases}\frac{\left|Q_{i j}\right|+\left|Q_{j i}\right|}{2}=\left|V_{i}\right|\left|V_{j}\right|\left|G_{i j} \cos \left(\phi_{i}-\phi_{j}\right)\right| & i \neq j \\ 0 & i=j\end{cases}$

where $V_{i}$ and $V_{j}$ are the voltage amplitudes of nodes $i$ and $j$, respectively; $G_{i j}$ is the real part of the admittance matrix; and $\phi_{i}, \phi_{j}$ are the phase angles between the voltage and the current at the nodes $i$ and $j$.

\subsection{Active power similarity matrix}

Similar to the reactive power, the minimum active power flow disruption, as shown in (9), can be defined and utilized for controlled islanding.

$\min _{V_{1}, V_{2} \subset V}\left(\sum_{i \in V_{1}, j \in V_{2}}\left|P_{i j}\right|\right)$

where $P_{i j}$ is the active power flow between bus $i$ and bus $j$. The controlled islanding problem with the above objective function can be similarly transformed to a graph-cut problem by defining a squared $N_{B} \times N_{B}$ adjacency matrix with elements $\left|P_{i j}\right|$. Accordingly, an active power graph similarity matrix $\boldsymbol{M}_{P}$ is defined as:

$M_{P, i j}= \begin{cases}\frac{\left|P_{i j}\right|+\left|P_{j i}\right|}{2}=\left|V_{i}\right|\left|V_{j}\right|\left|B_{i j} \sin \left(\phi_{i}-\phi_{j}\right)\right| & i \neq j \\ 0 & i=j\end{cases}$

where $B_{i j}$ is the imaginary part of the network admittance matrix.

Utilizing the minimum power flow disruption as the objective function minimizes the amount of load that must be shed following system splitting. The three proposed similarity matrics $\boldsymbol{M}_{C C S M}, \boldsymbol{M}_{Q}$ and $\boldsymbol{M}_{P}$ are calculated based on real-time power system data. When the aforementioned simlarity matrices are used in one model, it can be anticipated that an appropriate combination of information included in the multiple graph layers would lead to an improved clustering. This will lead to more precise predictions on the location and extension of the island of stability.

\section{Controlled islanding via multi-layer spectral clustering while addressing generator coherency}

\subsection{Stage I: coherency detection based on modularity clustering}

Based on the concept of tight coherency, the phase angles of all buses in an area should have relatively the same deviation. This can be assessed by calculating the correlation between each pair of buses in the area using (5).

To identify the coherency of buses, it is necessary to find strongly connected groups of buses since groups that are strongly coupled tend to maintain synchronism. Online coherency detection based on modularity clustering algorithm will be used to achieve this purpose. It requires neither a predefined number of groups nor a defining threshold value. The objective of this method is to separate the network into groups of vertices that have weak connections between them and to look for the naturally occurring groups in a network regardless of the number size. Greedy optimization of modularity tends to realize very fast clustering.

The modularity is defined as the number of edges falling within groups minus the expected number in an equivalent network with edges placed at random. The modularity, denoted by $Q$, is given by:

$Q=\frac{1}{2 m} \sum_{i j}\left(w_{i j}-\frac{d_{i} d_{j}}{2 m}\right) \delta\left(C_{i}, C_{j}\right)$

where $w_{i j}$ is the weight of the edge between $i$ and $j ; d_{i}$ and $d_{j}$ are the degrees of the vertices $i$ and $j$, respectively; $m$ is the total number of the edges; and $\delta$-function is 1 if nodes $i$ and $j$ are in the same community $\left(C_{i}=C_{j}\right)$, otherwise, it is 0 . The value of $Q$ lies in the range $[-1,1]$. The cluster structure can be searched precisely by checking the network divisions that have large modularity values.

The first step in evaluating coherency of buses in a power network at any point in time is to calculate the correlation coefficient among all the buses and form the correlation coefficient similarity matrix. Then $k$ groups of coherent buses can be achieved by applying modularity clustering on the correlation coefficient similarity matrix.

\subsection{Stage II: controlled islanding while preserving coherent bus groups}

In graph theory, spectral clustering treats the data clustering as a graph partitioning problem, which is equivalent 
to minimizing weights of graph cuts. Further, the normalized cuts algorithm can be used to find the solution to the normalized cuts problem. It substantially corresponds to working with the eigenvectors and eigenvalues of the normalized graph Laplacian. The normalized graph Laplacian matrix $\boldsymbol{L}$ is of broad interests in the studies of spectral graph theory and is defined as:

$\boldsymbol{L}=\boldsymbol{D}^{\frac{1}{2}}(\boldsymbol{D}-\boldsymbol{W}) \boldsymbol{D}^{-\frac{1}{2}}$

where $\boldsymbol{D}$ is the degree matrix, i.e., a diagonal matrix with the vertex degrees along the diagonal that are defined as $D_{i j}=\sum_{j=1}^{M} A_{i j}, A_{i j}$ is the component of the adjacency matrix $\boldsymbol{A}$ of $G$; and $\boldsymbol{W}$ is the adjacency matrix.

We consider now the problem of clustering $N_{B}$ vertices, $V=\left\{v_{i}, i=1,2, \ldots, N_{B}\right\}$ of $G$ into $k$ distinct subsets so that the bus nodes in the same subset are similar, i.e., they are connected by edges of large weights. Reference [20] proved that all normalized Laplacian eigenvalues of a graph lie in the interval $[0,2]$, and 0 is always a normalized Laplacian eigenvalue, a property favorable in comparing different graph layers. We note that the spectral clustering algorithms can efficiently solve this problem. Precisely, we concentrate on the algorithm suggested in [18], which solves the following trace minimization problem:

$\left\{\begin{array}{l}\min _{\boldsymbol{U} \in \mathbf{R}^{N_{B} \times k}} \operatorname{tr}\left(\boldsymbol{U}^{\mathrm{T}} \boldsymbol{L} \boldsymbol{U}\right) \\ \text { s.t. } \boldsymbol{U}^{\mathrm{T}} \boldsymbol{U}=\boldsymbol{I}\end{array}\right.$

where $\boldsymbol{U}$ is the spectral embedding matrix.

The clustering of the vertices in $G$ is then implemented using the $k$-means clustering algorithm to the normalized row vectors of the matrix $\boldsymbol{U}$.

Given a multi-layer graph $G$ with $M$ individual layers $\left\{G_{i}, i=1,2, \ldots, M\right\}$, we first compute the graph Laplacian matrix $\boldsymbol{L}_{i}$ for each $G_{i}$ and then represent each $G_{i}$ by the spectral embedding matrix $\boldsymbol{U}_{i} \in \mathbf{R}^{N_{B} \times k}$ from the first $k$ eigenvectors of $\boldsymbol{L}_{i}$.

The goal is to merge these multiple subspaces in a meaningful and efficient way. To merge these multiple subspaces, the Riemannian squared projection distance between the target representative subspace $\boldsymbol{U}$ and the $M$ individual subspaces $\left\{\boldsymbol{U}_{i}, i=1,2, \ldots, M\right\}$ is computed as the sum of the squared projection distances between $\boldsymbol{U}$ and each individual subspace given by $\boldsymbol{U}_{i}$ :

$d_{p r o j}^{2}=\sum_{i=1}^{M}\left(k-\operatorname{tr}\left(\boldsymbol{U} \boldsymbol{U}^{\mathrm{T}} \boldsymbol{U}_{i} \boldsymbol{U}_{i}^{\mathrm{T}}\right)\right)=k M-\sum_{i=1}^{M} \boldsymbol{U} \boldsymbol{U}^{\mathrm{T}} \boldsymbol{U}_{i} \boldsymbol{U}_{i}^{\mathrm{T}}$

By solving the following optimization problem that integrates both (13) and (14), multiple subspaces can be merged. This method is based on the following RayleighRitz theorem, which transforms the generalized eigenvalues problem into a constrained minimization problem, described as:

$\left\{\begin{array}{l}\min _{\boldsymbol{U} \in \mathbf{R}^{N_{B} \times k}} \operatorname{tr}\left(\boldsymbol{U}^{\mathrm{T}}\left(\sum_{i=1}^{M} \boldsymbol{L}_{i}-\alpha \sum_{i=1}^{M} \boldsymbol{U}_{i} \boldsymbol{U}_{i}^{\mathrm{T}}\right) \boldsymbol{U}\right) \\ \text { s.t. } \boldsymbol{U} \boldsymbol{U}^{\mathrm{T}}=\boldsymbol{I}\end{array}\right.$

where $\alpha$ is the weighting parameter that balances the tradeoff linking the two terms in the objective function. We may note that this is identical trace minimization problem as introduced in (13), but with a "modified" Laplacian given as:

$\boldsymbol{L}_{m}=\sum_{i=1}^{M} \boldsymbol{L}_{i}-\alpha \sum_{i=1}^{M} \boldsymbol{U}_{i} \boldsymbol{U}_{i}^{\mathrm{T}}$

The proposed M-SCCI algorithm is described as follows.

1) The first stage

Step 1: formulate the multi-layer graph $G$ using only bus nodes, with edge weights equal to the $C_{C, i j}, M_{P, i j}$ and $M_{Q, i j}$.

Step 2: obtain the $k$ cluster groups of coherent buses from Step 1.

2) The second stage

Step 3: input $N_{B} \times N_{B}$ weighted adjacency matrices $\left\{\boldsymbol{W}_{i}, i=1,2, \ldots, M\right\}$ of each individual graph layers $\left\{G_{i}, i=1,2, \ldots, M\right\}, k$, and $\alpha$.

Step 4: calculate the normalized Laplacian matrix $\boldsymbol{L}_{i}$ and the subspace illustration $\boldsymbol{U}_{i}$ for each $G_{i}$. (16).

Step 5: compute the graph Laplacian matrix $\boldsymbol{L}_{m}$ with

Step 6: compute $\boldsymbol{U} \in \mathbf{R}^{N_{B} \times k}$.

Step 7: normalize each row of $\boldsymbol{U}$ to get $\boldsymbol{U}_{\text {norm }}$.

Step 8: let $\boldsymbol{y}_{j} \in \mathbf{R}^{k}(j=1,2, \ldots, n)$ be the transpose of the $j^{\text {th }}$ row of $\boldsymbol{U}_{\text {norm }}$.

Step 9: cluster $\boldsymbol{y}_{j}$ into $C_{1}, C_{2}, \ldots, C_{k}$ using the $k$-means algorithm.

Step 10: output cluster assignments $C_{1}, C_{2}, \ldots, C_{k}$.

This algorithm uses the correlation coefficient between the frequency components among $C_{C, i j}, M_{P, i j}$, and $M_{Q, i j}$ data to produce an islanding solution with minimum power flow disruption. In the first stage, the buses are grouped using modularity clustering, based on the $C_{C, i j}$. The number of $k$-cluster outcomes in this grouping serves as the input to the second stage, in which nodes are grouped based on multi-layer constrained spectral clustering. The M-SCCI algorithm proposed here can identify, in real time, an islanding solution that has minimum power flow disruption and satisfies the bus coherency constraints. 


\section{Simulation studies}

The model effectiveness is evaluated through the simulation study conducted on the modified IEEE 39-bus system. The methodology has been implemented in MATLAB and all time-domain simulations are achieved in DIgSILENT PowerFactory. To stress the system and raise the likelihood of instability following a disturbance, we increase the base load level by $25 \%$ at $0.01 \mathrm{~s}$. Then, two short circuit events occur in lines 13-14 and 16-17 at $2 \mathrm{~s}$. The short circuit events are cleared after $0.20 \mathrm{~s}$ by opening the line switches from the substations, while the simulation lasts for $5 \mathrm{~s}$.

Figures 2 and 3 demonstrate the rotor angles of generators and the system frequencies, respectively, which indicate the system instability following the short circuit events. The proposed solution approach is applied to the system to determine the islanding boundaries. The quality of each island is then evaluated by calculating the dynamic behavior and the power mismatch in the islands. It can be observed from Fig. 3 that if no control action is undertaken, the system loses synchronism at about $2.25 \mathrm{~s}$. Indeed, real-time simulation in DIgSILENT indicates out of step at $2.25 \mathrm{~s}$ for generators. As noticed, the system is divided into two groups, which are not balanced.

The frequency of the generators and the loss of synchronism are a clear indication that the system should be split.

Before proceeding to discuss these case studies for our proposed methodology, we examine the islanding methodology proposed in [9] to split the network. In this method, the authors proposed a two-step constrained spectral clustering-controlled islanding to find the islanding solution, which provided the minimum power flow disruption while satisfying the constraint of coherent generator groups.

As it will be shown, in the following example, a multiple variant of valid cut-sets separating coherent generator groups from each other is possible, but only certain variants will allow secure islanding.

According to the proposed model in [9], it is essential to find the minimum cut in a graph that its edges are the active

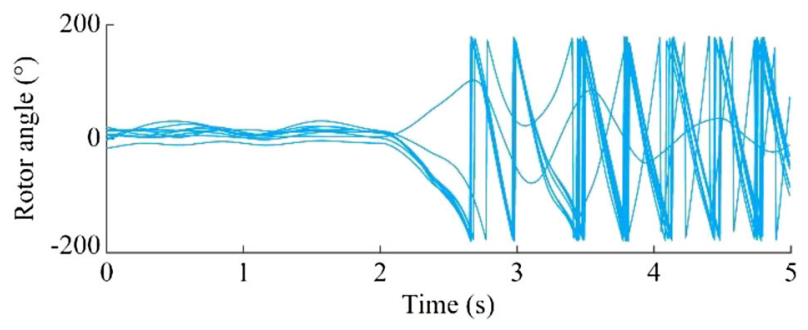

Fig. 2 Rotor angles following two short circuit events without islanding

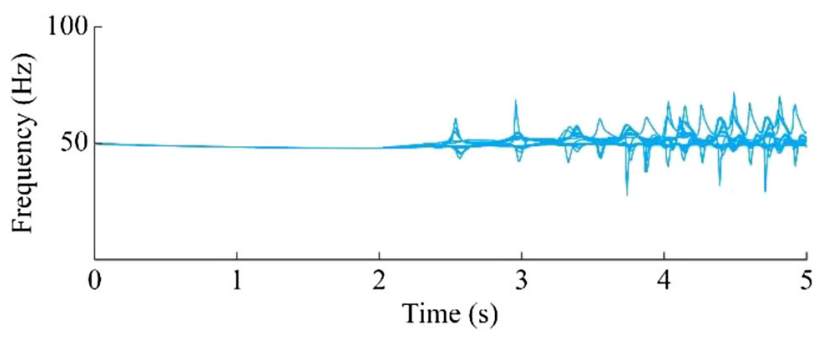

Fig. 3 System frequencies following two short circuit events without islanding

power distortion and constraint the clusters with the coherent groups of generators. Accordingly, we need to firstly find the coherent groups of generators and then establish the connectivity constraints among the generators within a group and non-connectivity constraints between the generators in different groups. Finally, the spectral constraint clustering is applied to the problem and determine the islands in the power system.

Following clearing the fault and applying the modularity clustering to the $\boldsymbol{K}_{\mathrm{s}}$ matrix proposed in [16] at $2.21 \mathrm{~s}$, two coherent groups of generators, $\{\mathrm{G} 1, \mathrm{G} 2, \mathrm{G} 3, \mathrm{G} 8, \mathrm{G} 9, \mathrm{G} 10\}$ and $\{\mathrm{G} 4, \mathrm{G} 5, \mathrm{G} 6, \mathrm{G} 7\}$, are produced. The two coherent groups of generators form the set of connectivity and nonconnectivity constraints, in which all pairs of generators in one group must be linked together (connectivity constraints), and generators in different groups must not be linked with each other (non-connectivity constraints), where the associated schematic is shown in Fig. 4.

After determining the coherent groups of generators, the active power graph similarity matrix is clustered into two groups, using the constraint clustering approach [21], where the outcome of clusters and the rotor angles of generators following the clustering are depicted in Figs. 5 and 6 , respectively.

As can be seen, while the generators in island 2 are stable, generators in island 1 become out of steps. On the

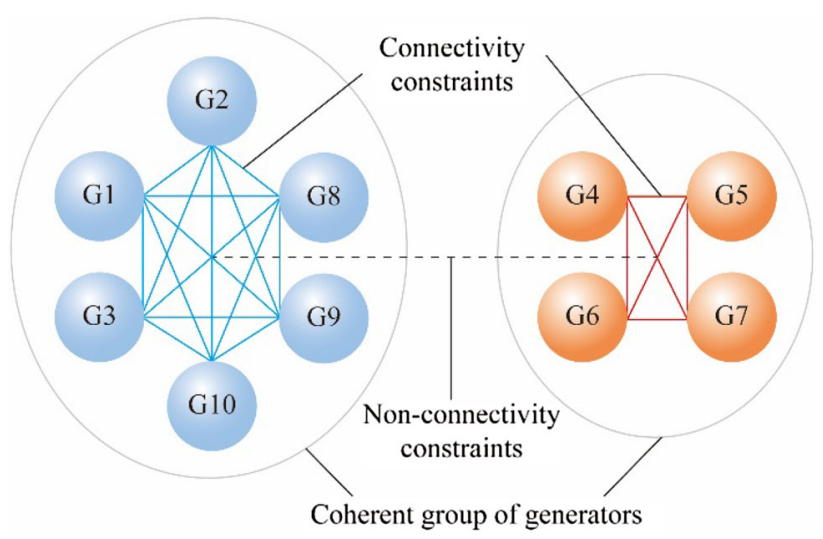

Fig. 4 Coherent groups of generators and constraints of connectivity and non-connectivity 
other hand, the graph-based islanding solution automatically satisfies the connectivity constraints, since the solution is sought through the minimum cuts in the graph. Our approach is a graph-based approach, wherein in each island the nodes preserve their pre-islanding conditions, and the network is separated by cutting the edges in the graph.

The following sub-sections compare the result using three different criteria, i.e. frequency similarity, reactive power, and active power, for the islanding decision making procedure. Four cases in the same operating conditions are employed to demonstrate the accuracy of the proposed M-SCCI algorithm.

Case 1: single-layer intentional islanding based on frequency similarity of the island's buses.

Case 2: single-layer intentional islanding based on reactive power.

Case 3: single-layer intentional islanding based on active power.

Case 4: multi-layer intentional islanding based on all three criteria.

In all cases, the islanding scheme is applied at $2.21 \mathrm{~s}$, just after clearing the fault to avoid generator instability that happens at $2.25 \mathrm{~s}$ if no action is taken.

\subsection{Case 1}

In this case study, the frequency similarity is employed as the main criterion for islanding decision making. The approach provides a suitable islanding solution using online coherency and pre-fault power flow conditions. In the first stage, the proposed buses coherency modularity clustering algorithm based on frequency similarity of the island's buses identified two sets of coherent generators $\{\mathrm{G} 1, \mathrm{G} 2, \mathrm{G} 3, \mathrm{G} 10\}$ and $\{\mathrm{G} 4, \mathrm{G} 5, \mathrm{G} 6, \mathrm{G} 7, \mathrm{G} 8, \mathrm{G} 9\}$.

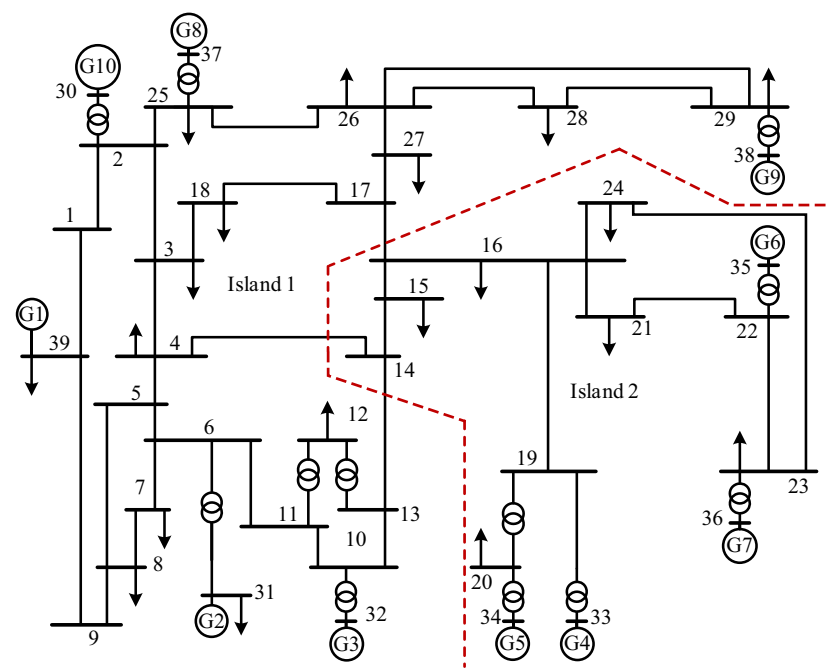

Fig. 5 Islanding boundaries after applying clustering method

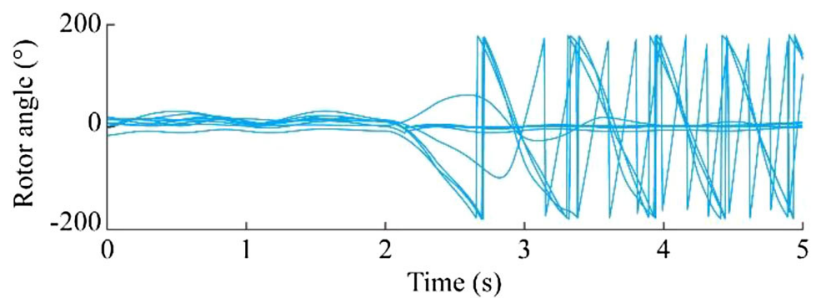

Fig. 6 Rotor angles of generators after applying clustering method

Considering these two sets found in first stage, the number of two clusters outcomes serves as the input in the second stage to solve the single-layer constrained spectral clustering. The islanding solution suggests that it should be split into two islands as shown in Fig. 7. The resulted groups using $C_{C, i j}$ are two groups as depicted in Fig. 8 with two background colors.

The allocation of buses in Case 1 to coherent generator groups is as follows: (1) island 1, buses B1-B14, B30-B32, and B39; (2) island 2, buses B15-B29 and B33-B38. The minimum power flow disruption across boundaries of islands is $857 \mathrm{MW}$ active power and 1349 Mvar reactive power.

Figure 9 shows the generator rotor angle oscillations during the simulation study of $15 \mathrm{~s}$. Obviously, the rotor angle oscillations are unstable, and all the machines lose synchronism while groups of generators become weaker following the events.

\subsection{Case 2}

In this case study, the minimum reactive power flow disruption is employed as the main criterion for islanding

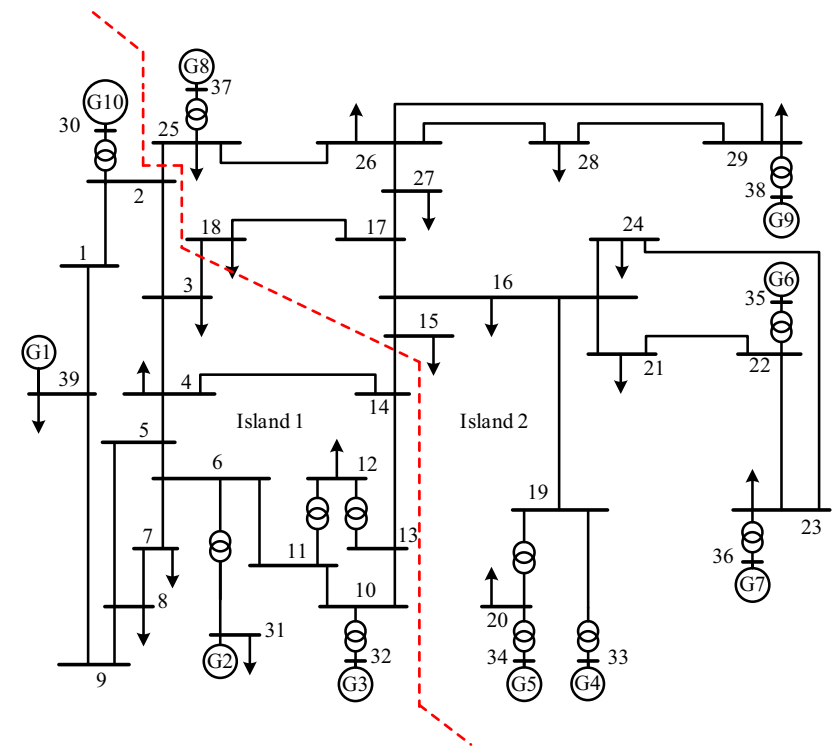

Fig. 7 Islanding boundaries considering frequency similarity 


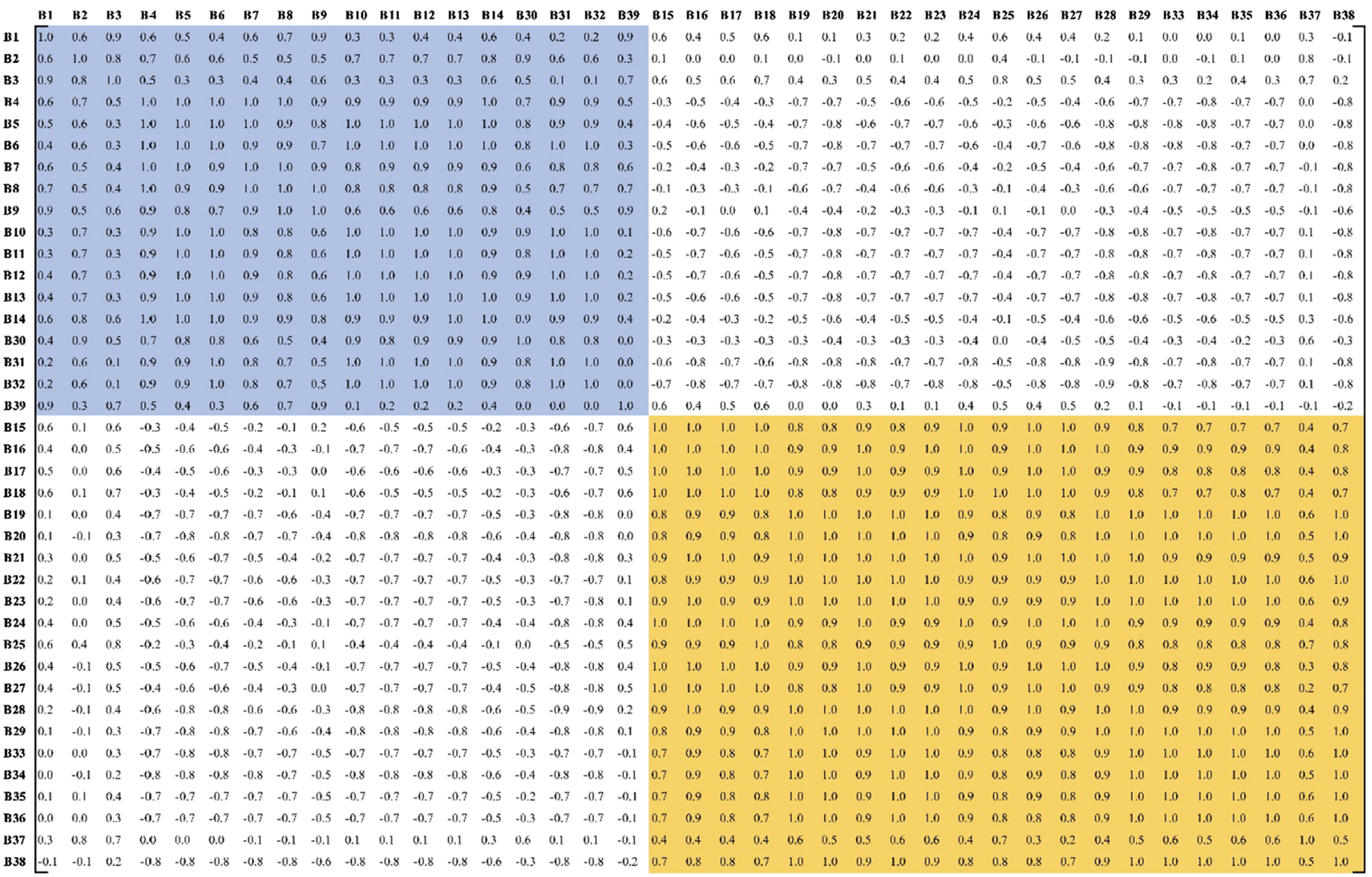

Fig. 8 Correlation coefficient similarity matrix at $2.21 \mathrm{~s}$

decision making procedure. The system initial condition is the same as that of Case 1. The same faults as that of Case 1 are imposed. In the first stage of the proposed bus coherency, modularity clustering algorithm based on reactive power similarity of the island's buses identified three sets of coherent generators $\{\mathrm{G} 1, \mathrm{G} 2, \mathrm{G} 3, \mathrm{G} 10\},\{\mathrm{G} 4$, G5, G6, G7 \}, and $\{\mathrm{G} 8, \mathrm{G} 9\}$.

Considering these three sets found in the first stage, the number of outcomes of three clusters serves as the input in the second stage to solve the single-layer constrained spectral clustering based on the minimum reactive power flow disruption. The islanding solution suggests that it should be three islands as shown in Fig. 10. The resulted groups using $M_{Q, i j}$ are three groups as depicted in Fig. 11 with three background colors.

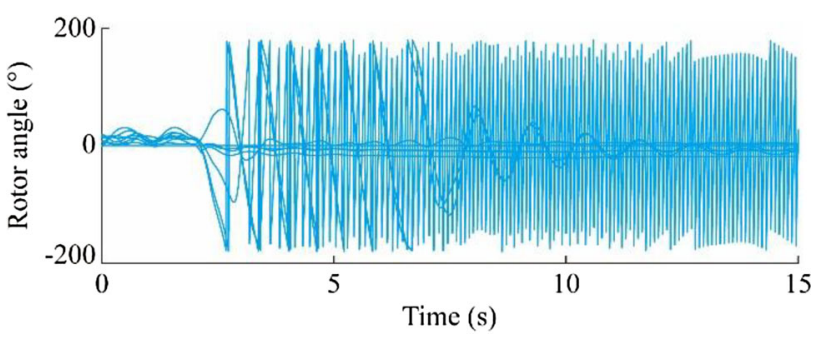

Fig. 9 Rotor angles after islanding based on frequency similarity
The allocation of buses in Case 2 to coherent generator groups is as follows: (1) island 1, buses B1-B15, B18, B30-B32, and B39; (2) island 2, buses B16, B19-B24, and B33-B36; (3) island 3, buses B17, B25-B29, B37, and B38. The minimum power flow disruption across boundaries of islands is $2291 \mathrm{MW}$ active power and 1349 Mvar reactive power.

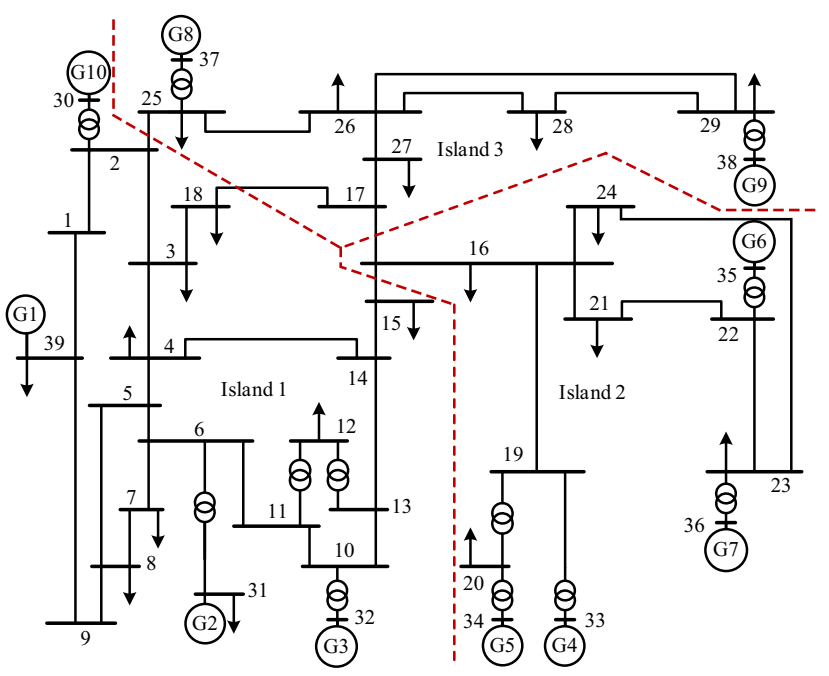

Fig. 10 Islanding boundaries considering reactive power 
Figure 12 shows the generator rotor angle oscillations during the simulation study of $15 \mathrm{~s}$. Obviously, the rotor angle oscillations are damped, and all the machines lose synchronism while groups of generators become weaker following the events.

\subsection{Case 3}

In this case study, the minimum active power flow disruption is employed as the main criterion for islanding decision making procedure. The system initial condition and the fault are the same as that of Case 1 .

The first stage of the proposed bus coherency modularity clustering algorithm based on active power similarity of the island's buses returnes three coherent generator groups $\{\mathrm{G} 1, \mathrm{G} 2, \mathrm{G} 3, \mathrm{G} 8, \mathrm{G} 10\},\{\mathrm{G} 4, \mathrm{G} 5, \mathrm{G} 6, \mathrm{G} 7\}$, and $\{\mathrm{G} 9\}$.

Considering these three sets found in the first stage, the number of outcomes of three clusters serves as the input in the second stage to solve the single-layer constrained spectral clustering based on the minimum active power flow disruption. The resulted groups using the $M_{P, i j}$ are three groups as depicted in Fig. 13 with three background colors. The islanding solution suggests that there should be three islands as shown in Fig. 14.

The allocation of buses in Case 3 to coherent generator groups is as follows: (1) island 1, buses B1-B15, B25, B30$\mathrm{B} 32$, and $\mathrm{B} 39$; (2) island 2, buses B16-B24 and B33-B36; (3) island 3, buses B26-B29 and B38. The minimum power flow disruption across boundaries of islands is $1108 \mathrm{MW}$

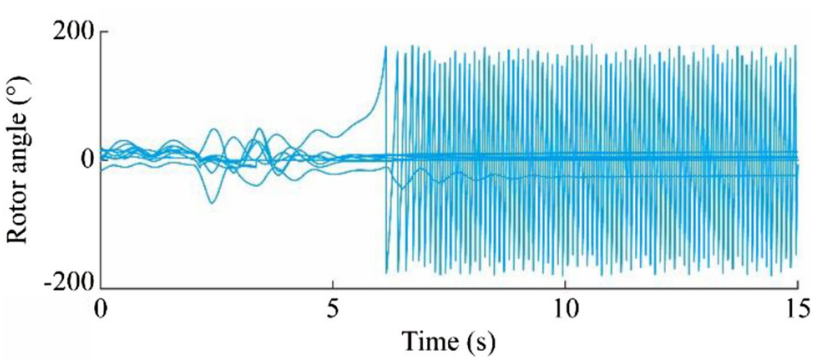

Fig. 12 Rotor angles after islanding based on reactive power

active power and 1349 Mvar reactive power are disrupted.

Figure 15 shows the generator rotor angle oscillations during the simulation study of $15 \mathrm{~s}$. Obviously, the rotor angle oscillations are damped, and all the machines lose synchronism. As can be seen, the power system is not stable.

\subsection{Case 4}

The system initial condition and the fault are the same as all the previous cases. In this case study, the frequency, active power, and reactive power similarity matrices are employed as the main criteria for islanding decision making procedure. We implemented intentional islanding at $2.21 \mathrm{~s}$ following two cascading outages. First, correlation coefficient is calculated using (5) for all pairs of buses which result in the $C_{C, i j}$ shown in Fig. 8. Applying

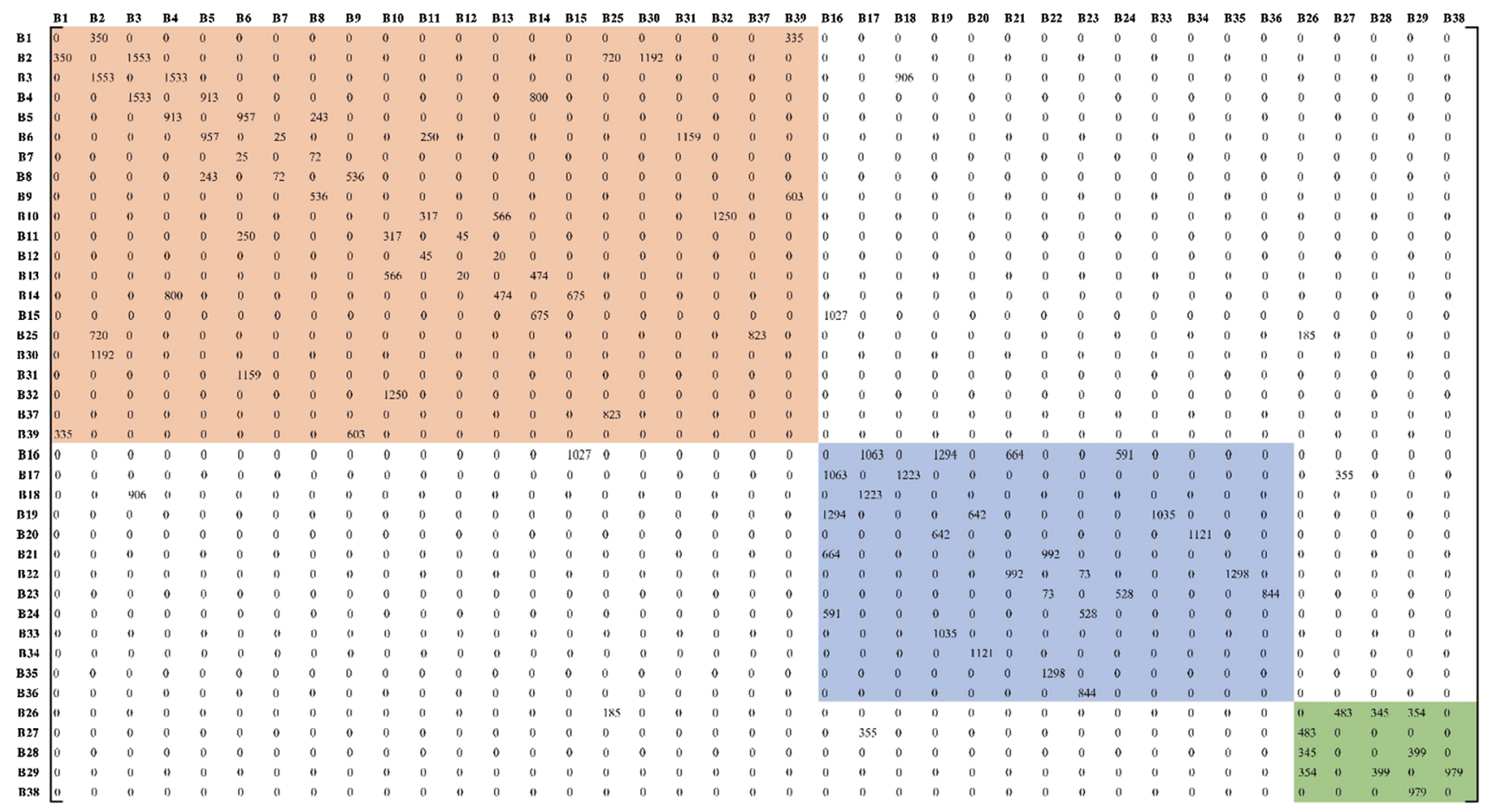

Fig. 11 Reactive power similarity matrix at $2.21 \mathrm{~s}$ 


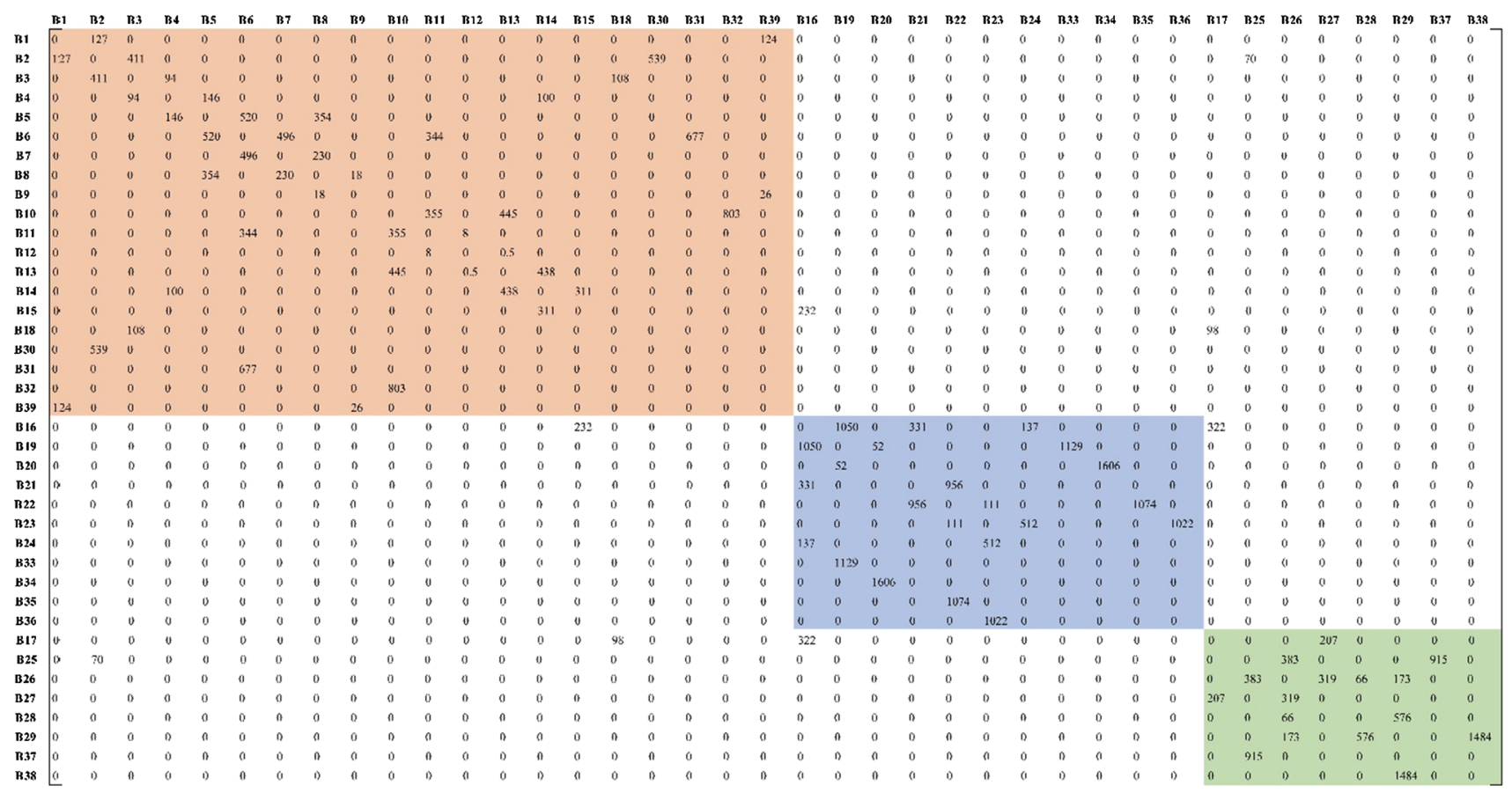

Fig. 13 Active power similarity matrix at $2.21 \mathrm{~s}$

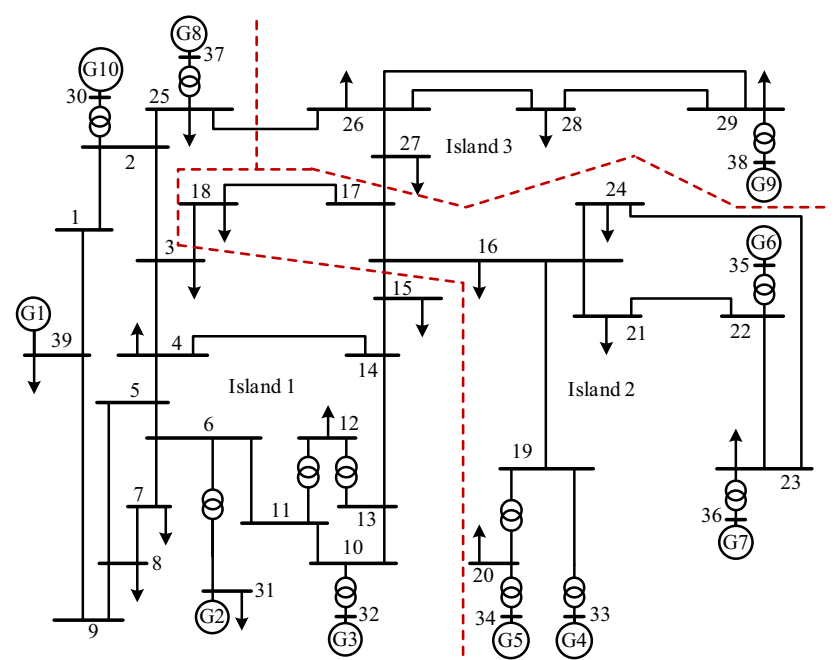

Fig. 14 Islanding boundaries considering active power

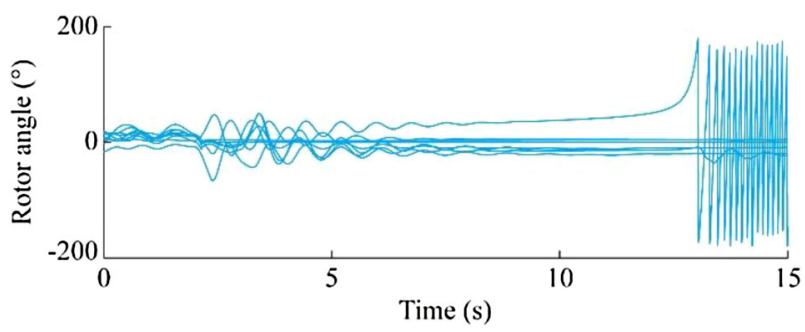

Fig. 15 Rotor angles after islanding based on active power the modularity clustering on the $C_{C, i j}$ returned two coherent generator groups $\{\mathrm{G} 1, \mathrm{G} 2, \mathrm{G} 3, \mathrm{G} 8, \mathrm{G} 9, \mathrm{G} 10\}$ and $\{\mathrm{G} 4, \mathrm{G} 5, \mathrm{G} 6, \mathrm{G} 7\}$.

Considering these two sets found in the first stage, the number of two clusters outcomes serves as the input in the second stage to solve the M-SCCI. Then, in the second stage, the M-SCCI algorithm is excused using the threelayer graph with weighted adjacency matrices, i.e. $\boldsymbol{M}_{C C S M}$, $\boldsymbol{M}_{Q}$ and $\boldsymbol{M}_{P}$, as the main criteria for islanding decision making procedure, taking into consideration the two cluster coherency groups found in the first stage of the algorithm. The allocation of buses in Case 4 to the coherent generator groups is as follows: (1) island 1, buses B1-B14, B17, B18, B25-B32, and B37-B39; (2) island 2, buses B15, B16, B19B24, and B33-B36.

The final splitting strategy possessing the lowest power exchange is represented in Fig. 16. The minimum power flow disruption across boundaries of islands is $622 \mathrm{MW}$ active power and 1349 Mvar reactive power. Figure 17 shows the generator rotor angle oscillations during the simulation study of $15 \mathrm{~s}$. Obviously, the rotor angle oscillations are damped, and all the machines remain in synchronism while groups of generators become stronger following the events. 


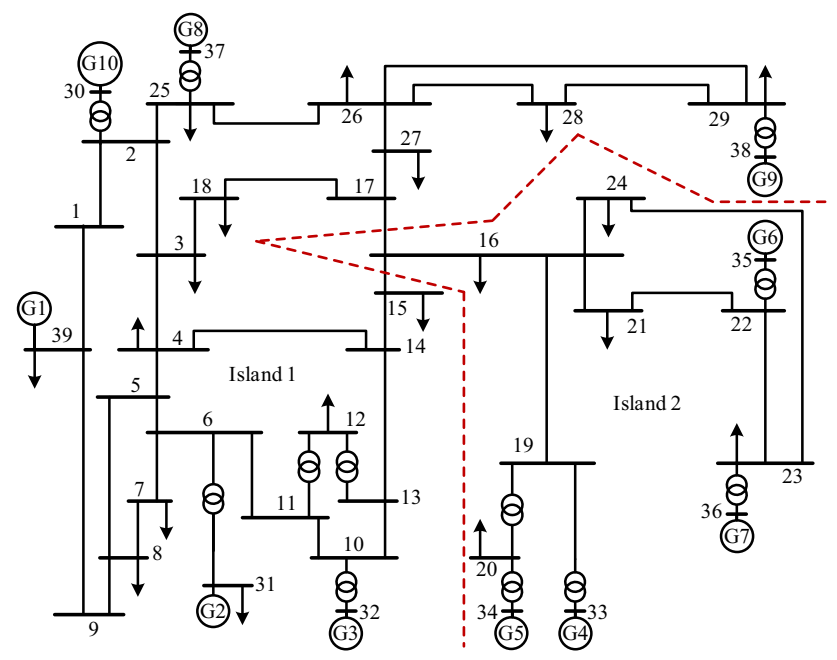

Fig. 16 Islanding boundaries based on multi-layer clustering

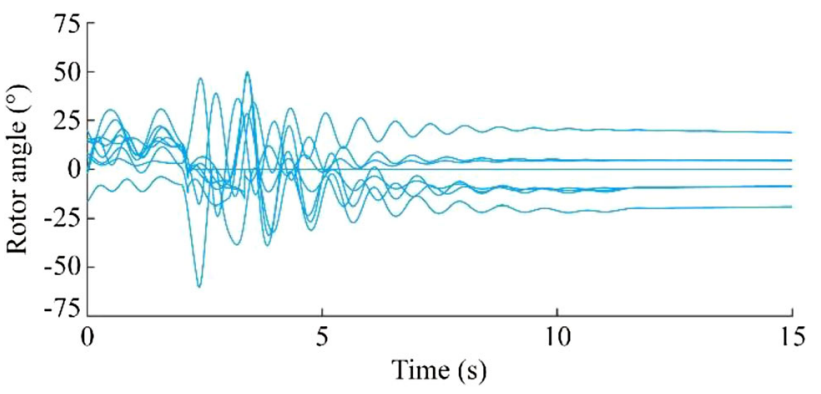

Fig. 17 Rotor angles after islanding based on all criteria
Table 1 presents the power flow mismatch between the islands for each case study, where $P_{G}$ and $Q_{G}$ are the active and reactive power of generator, $P_{L}$ and $Q_{L}$ are the active and reactive power of load, $\Delta P=P_{G}-P_{L}$, and $Q=Q_{G}-Q_{L}$. The comparison in Table 1 shows that the proposed M-SCCI algorithm using all criteria returns the cut-set that separates the coherent generator groups with minimum cut, which is $622 \mathrm{MW}$.

\section{Conclusion}

This paper propose a computationally efficient real-time ICI algorithm based on multi-layer graphs, subspace analysis, and constrained spectral clustering while addressing the generator coherency problem. We demonstrate that using multi-layer spectral clustering to find the islanding boundaries, instead of using a single layer, i.e., the frequency similarity, the active power, and the reactive power produced improves clustering performance. The insertion of the bus coherency constraints prevents new island groupings that would contain non-coherent generators. The use of minimum power-flow disruption improves the transient stability of the islands produced. The simulation results show that the proposed M-SCCI algorithm is computationally efficient and is suitable for using in realtime applications involving large power systems.

Table 1 Summary of power flow mismatch between islands for each case study

\begin{tabular}{|c|c|c|c|c|c|c|c|c|c|}
\hline Clustering criteria & Island & $P_{G}(\mathrm{MW})$ & $P_{L}(\mathrm{MW})$ & $Q_{G}($ Mvar $)$ & $Q_{L}(\mathrm{Mvar})$ & $\begin{array}{l}\Delta P \\
(\mathrm{MW})\end{array}$ & $\begin{array}{l}\Delta Q \\
(\text { Mvar })\end{array}$ & $\begin{array}{l}\sum_{(\mathrm{MW})}|\Delta P| \\
\end{array}$ & $\begin{array}{l}\sum_{\text {(Mvar) }}|\Delta Q| \\
\text { (Mval }\end{array}$ \\
\hline \multirow{3}{*}{$\begin{array}{l}\text { Clustering based on reactive } \\
\text { power }\end{array}$} & 1 & 3672 & 4765 & 1663 & 1458 & -1093 & 205 & \multirow[t]{3}{*}{2291} & \multirow[t]{3}{*}{1349} \\
\hline & 2 & 3525 & 2681 & 1397 & 364 & 844 & 1032 & & \\
\hline & 3 & 2055 & 1700 & 402 & 291 & 355 & 111 & & \\
\hline \multirow[t]{3}{*}{ Clustering based on active power } & 1 & 4482 & 4864 & 1802 & 1484 & -381 & 318 & \multirow[t]{3}{*}{1107} & \multirow[t]{3}{*}{1349} \\
\hline & 2 & 3525 & 2918 & 1397 & 409 & 607 & 987 & & \\
\hline & 3 & 1245 & 1364 & 264 & 221 & -119 & 44 & & \\
\hline \multirow{2}{*}{$\begin{array}{l}\text { Clustering based on frequency } \\
\text { similarity }\end{array}$} & 1 & 5580 & 5098 & 1799 & 930 & 482 & 869 & \multirow[t]{2}{*}{857} & \multirow[t]{2}{*}{1349} \\
\hline & 2 & 3672 & 4048 & 1663 & 1183 & -375 & 480 & & \\
\hline \multirow{3}{*}{$\begin{array}{l}\text { Clustering based on all three } \\
\text { criteria, } k=3\end{array}$} & 1 & 2055 & 1700 & 402 & 291 & 355 & 111 & \multirow[t]{3}{*}{1331} & \multirow[t]{3}{*}{1349} \\
\hline & 2 & 3525 & 3161 & 1397 & 594 & 364 & 803 & & \\
\hline & 3 & 3672 & 4285 & 1663 & 1228 & -612 & 435 & & \\
\hline \multirow{2}{*}{$\begin{array}{l}\text { Clustering based on all three } \\
\text { criteria, } k=2\end{array}$} & 1 & 5727 & 5985 & 2066 & 1520 & -258 & 546 & \multirow[t]{2}{*}{622} & \multirow[t]{2}{*}{1349} \\
\hline & 2 & 3525 & 3161 & 1397 & 594 & 364 & 803 & & \\
\hline
\end{tabular}


Open Access This article is distributed under the terms of the Creative Commons Attribution 4.0 International License (http:// creativecommons.org/licenses/by/4.0/), which permits unrestricted use, distribution, and reproduction in any medium, provided you give appropriate credit to the original author(s) and the source, provide a link to the Creative Commons license, and indicate if changes were made.

\section{References}

[1] Trodden PA, Bukhsh WA, Grothey A et al (2014) Optimizationbased islanding of power networks using piecewise linear AC power flow. IEEE Trans Power Syst 29(3):1212-1220

[2] Sun K, Zheng D, Lu Q (2003) Splitting strategies for islanding operation of large-scale power systems using OBDD-based methods. IEEE Trans Power Syst 18(2):912-923

[3] Ding T, Sun K, Huang C et al (2018) Mixed-integer linear programming-based splitting strategies for power system islanding operation considering network connectivity. IEEE Syst J 12(1):350-359

[4] Trodden P, Bukhsh W, Grothey A et al (2013) MILP formulation for controlled islanding of power networks. Int $\mathrm{J}$ Electr Power Energy Syst 45(1):501-508

[5] Kamali S, Amraee T (2017) Blackout prediction in interconnected electric energy systems considering generation re-dispatch and energy curtailment. Appl Energy 187:50-61

[6] Kamali S, Amraee T, Capitanescu F (2018) Controlled network splitting considering transient stability constraints. IET Gener Transm Distrib 12:5639-5648

[7] Ding L, Ma Z, Wall P et al (2017) Graph spectra based controlled islanding for low inertia power systems. IEEE Trans Power Del 32(1):302-309

[8] Chong Z, Dai Z, Wang S et al (2014) The application of binary particle swarm optimization in power restoration. In: Proceedings of 10th international conference on natural computation (ICNC), Xiamen, China, 19-21 August 2014, pp 349-353

[9] Ding L, Gonzalez-Longatt FM, Wall P et al (2013) Two-step spectral clustering controlled islanding algorithm. IEEE Trans Power Syst 28(1):75-84

[10] Esmaeilian A, Kezunovic M (2016) Controlled islanding to prevent cascade outages using constrained spectral k-embedded clustering. In: Proceedings of power systems computation conference (PSCC), Genoa, Italy, 20-24 June 2016, 6 pp

[11] Quirós-Tortós J, Wall P, Ding L et al (2014) Determination of sectionalising strategies for parallel power system restoration: a spectral clustering-based methodology. Electr Power Syst Res 116:381-390

[12] Khalil AM, Iravani R (2016) A dynamic coherency identification method based on frequency deviation signals. IEEE Trans Power Syst 31(3):1779-1787

[13] Shao H (2016) Adaptive three-stage controlled islanding to prevent imminent wide-area blackouts. Dissertation, Durham University

[14] Davarikia H, Znidi F, Aghamohammadi MR et al (2016) Identification of coherent groups of generators based on synchronization coefficient. In: Proceedings of IEEE PES general meeting (PESGM), Boston, USA, 17-21 July 2016, 5 pp

[15] Znidi F, Davarikia H, Iqbal K (2017) Modularity clustering based detection of coherent groups of generators with generator integrity indices. In: Proceedings of IEEE PES general meeting (PESGM), Chicago, USA, 16-20 July 2017, 5 pp

[16] Davarikia H, Barati M, Znidi F et al (2018) Real-time integrity indices in power grid: a synchronization coefficient based clustering approach. https://arxiv.org/abs/1804.02793. Accessed 9 April 2018

[17] Dong X, Frossard P, Vandergheynst P et al (2014) Clustering on multi-layer graphs via subspace analysis on Grassmann manifolds. IEEE Trans Signal Process 62(4):905-918

[18] Dong X, Frossard P, Vandergheynst P et al (2012) Clustering with multi-layer graphs: a spectral perspective. IEEE Trans Signal Process 60(11):5820-5831

[19] Jonsson M, Begovic M, Daalder J (2004) A new method suitable for real-time generator coherency determination. IEEE Trans Power Syst 19(3):1473-1482

[20] Chung FR, Graham FC (1997) Spectral graph theory. American Mathematical Society, Providence

[21] Rangapuram SS, Hein M (2012) Constrained 1-spectral clustering. In: Proceedings of the 15th international conference on artificial intelligence and statistics (AISTATS), La Palma, Canary Islands, 21-23 April 2012, 12 pp

Faycal ZNIDI received the B.S. and M.S. degrees in electrical engineering from University of Tennessee at Chattanooga, USA, in 2009 and 2011, respectively, and received the Ph.D. degree in engineering science and systems at University of Arkansas at Little Rock, USA, in 2019. His research interests include advanced optimization, operation research, microgrid operation and planning, and power system protection.

Hamzeh DAVARIKIA received the Ph.D. degree in electrical engineering from the Louisiana State University, USA, in 2019. Currently, he is an assistant professor at McNeese State University, USA. His research interests include operation research, optimization in power networks, power system dynamics, microgrid operation, and power system protection.

Kamran IQBAL received the M.S. and Ph.D. degrees in electrical engineering from the Ohio State University, USA. He is a professor of systems engineering in the College of Engineering and Information Technology at University of Arkansas at Little Rock, USA. His research interests include biomedical signal processing, electrical energy, and machine learning.

Masoud BARATI received the Ph.D. degree in electrical engineering from Illinois Institute of Technology, Chicago, USA, in 2013. Presently, he is an assistant professor in the Electrical and Computer Engineering Department at the University of Pittsburgh, Pittsburgh, USA. His research interests include advanced optimization, microgrid operation and planning, power system protection, microeconomics, mathematical modeling, and multiple infrastructure assessment. 